\title{
Incidental Diagnosis of Granular Cell Tumor of Vulva: Importance of Discussing Vulvar Health at the Annual Well- Woman Examination
}

\author{
Navleen Gilla, Mili Thakur $^{\mathrm{a}, \mathrm{b}}$, Jocelyn Peters ${ }^{\mathrm{a}}$, Awoniyi O. Awonuga ${ }^{\mathrm{a}}$
}

\begin{abstract}
Granular cell tumors are rare tumors of neurogenic or Schwann cell origin and are known to involve the vulva. The objective of this case is to highlight the importance of careful vulvar examination during the annual well-woman examination by presenting a case of an incidental finding of granular cell tumor of the vulva. A 55-year-old African American female was noted to have an incidental finding of a $2 \times 2 \mathrm{~cm}$ slow growing right vulvar lump at her yearly gynecologic exam. The lesion was excised and the histopathology was consistent with a benign granular cell tumor of the vulva. Immunostaining was positive for S100 and neuron-specific enolase. Asymptomatic vulvar masses can be the only presenting feature of a neoplastic process. Women may not seek medical attention due to embarrassment or lack of recognizing the importance of symptoms. This case report highlights such a scenario as a reminder to carefully examine the vulva during annual well-woman examination. A low threshold for biopsy can assist in diagnosing pre-invasive and invasive vulvar lesions.
\end{abstract}

Keywords: Granular cell tumor; Vulvar neoplasm; Vulvar tumor; Vulva

\section{Introduction}

The well-woman visit (annual health assessment) provides a unique opportunity for the health care provider to recognize risk factors for disease and identify inconspicuous disorders [1]. Careful examination of the vulva forms an integral part of this assessment and can identify indolent vulvovaginal disease. Changes in the vulva are often subtle, difficult to recognize by the patient and can be overlooked [2]. Discussion of

Manuscript accepted for publication August 04, 2015

aDepartment of Obstetrics and Gynecology, Detroit Medical Center/Wayne State University, Detroit, MI, USA

${ }^{\mathrm{b}}$ Corresponding Author: Mili Thakur, Division of Reproductive Endocrinology and Infertility, Wayne State University School of Medicine/Detroit Medical Center, 3990 John R. Street, Box 158, Detroit, MI 48201, USA.

Email: mthakur@med.wayne.edu

doi: http://dx.doi.org/10.14740/jmc2258w vulvar health, a thorough search for vulvar abnormalities and a low threshold for vulvar biopsy, is important in preventing diagnostic delays of important vulvar disease such as vulvar cancers [3]. We present herein a case of granular cell tumor of the vulva, a rare neoplastic lesion to highlight the importance of careful vulvar inspection during the annual well-woman examination.

\section{Case Report}

A 55-year-old African American multiparous woman presented for her yearly gynecologic exam. She had no complaints. Vulvar examination revealed a firm, non-tender, well-demarcated $2.5 \times 2 \mathrm{~cm}$ nodular mass in the lower third of the right labium majus and adjacent perineal skin. There was no inguinal lymphadenopathy. On further questioning, she reported that she first noticed the lump 1 year prior and although it had been slowly increasing in size, it did not bother her; hence she did not seek medical help. Due to the large size of the lesion, decision was taken to excise the mass in the operating room. Under general anesthesia, the mass was excised through a 4-cm elliptical incision that included a wide margin away from the firm tumor. Excision revealed a round, well-formed, firm, granular, light-pink mass. Her post-operative course and follow-up were uneventful. Histopathology demonstrated a benign granular cell tumor of the vulva. The tumor was moderate to highly cellular, composed of oval to polygonal-shaped cells with abundant coarsely granular eosinophilic cytoplasm (Fig. 1A, B). Tumor cells grew in infiltrating nests, cords, and trabeculae. No atypical features, including mitotic figures, nuclear pleomorphism or necrosis were appreciated (Fig. 1A, B). Immunostaining was positive for S100 (Fig. 1C) and neuronspecific enolase (Fig. 1D). The diagnosis was consistent with a benign granular cell tumor of the vulva.

\section{Discussion}

Our case highlights the importance of careful vulvar inspection during the annual well-woman examination. Vulvar preinvasive and invasive lesions are relatively uncommon but have a significant impact on women's health [4]. While some of these lesions are asymptomatic and indeed when sympto- 


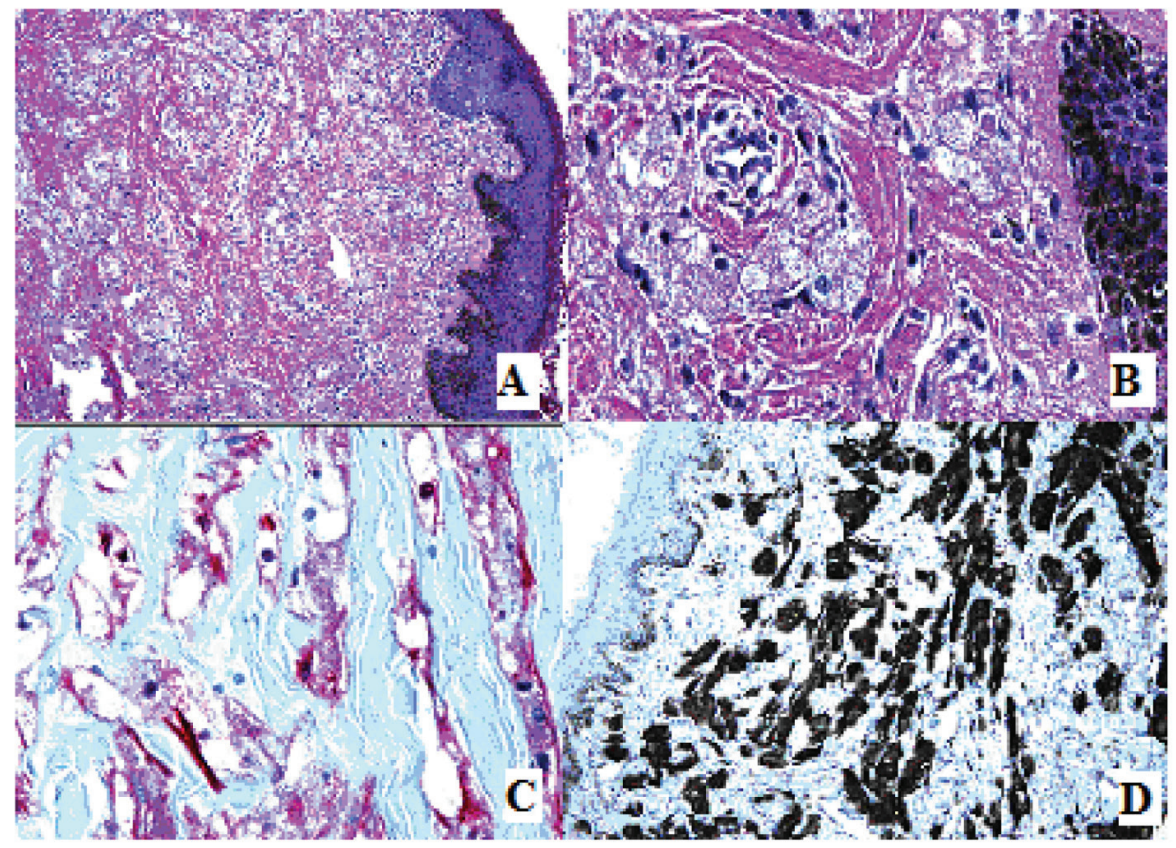

Figure 1. (A) Hematoxylin and eosin. Stratified squamous keratinized epithelium of the vulva is visualized with infiltrating tumor cells growing in nests, cords, and trabeculae. (B). Hematoxylin and eosin. Tumor cells are oval to polygonal-shaped with abundant coarsely granular eosinophilic cytoplasm. (C) S100 immunostaining with red chromogen. (D) Neuron-specific enolase immunostain.

matic, some patients may not recognize the importance of the symptoms and therefore not seek medical attention. Still, some may not report the symptoms due to embarrassment. There is also evidence to suggest that delay in diagnosis could be from failures or procrastination in performance of biopsies of abnormal appearing vulvar skin [3]. One study reported that $31 \%$ of women had three or more medical consultations for vulvar symptoms prior to the diagnosis of cancer [3].

Granular cell tumors are rare tumors of neurogenic or Schwann cell origin and hence can present in virtually any tissue with nerve cells. Accordingly, they have been reported in virtually all organs of the body including the head and neck region, with up to $15 \%$ occurring in the vulvar area [5]. They occur most commonly in the third to sixth decades of life. Although the etiology is unclear, females are predisposed more than males and occurrence is twice as often in African Americans $[6,7]$. In addition to being poorly understood, they present as nodules that are often palpable on the surface of the skin. Majority of these tumors are benign; however, about $1-2 \%$ are malignant [5].

As depicted by our case, benign granular cell tumors are usually small $(<3 \mathrm{~cm})$, and superficial, with typical macroscopic and histologic features. Most lesions are well circumscribed, but up to $50 \%$ may show poorly defined margins [5]. Granular cell tumors can mimic most known vulva cystic (Bartholin's gland and sebaceous cysts tumors) and solid benign painless lesions (fibromas, lipomas, papillomas and hidradenomas). However, unlike Bartholin's cyst that has a specific vulva anatomic position, granular cell tumor can occur anywhere in the vulva. Aside from the histological features described above, the granularity of the cytoplasm is typical in these tumors and is caused by the accumulation of phagolysosomes [6]. Secondary to the neurogenic origin, immunohistochemical staining is positive for S100, neuron-specific enolase, peripheral nerve myelin proteins and vimentin.

There is no established standard of care for granular cell tumors. However, because of the potential for recurrence and indeed malignancy, the current recommendation is wide local excision with clear margins [6]. Reports of the rate of recurrence of granular cell tumors vary anywhere from $2 \%$ to $10 \%$ $[5,6]$. Local recurrence usually occurs within 2 years and is likely a result of inadequate excision of the primary lesion. Our patient has been followed for 15 months without recurrence and if the granular cell tumor does not reoccur in the next 1 year, it would validate wide local excision as the treatment of choice in granular cell tumor. Repeated recurrence despite benign histological features, however, can be indicative of potential malignant behavior. Exclusion of malignancy depends on the absence of six histopathologic criteria proposed by Fanburg Smith and colleagues [7] namely: 1) the presence of necrosis, 2) the emergence of spindle cells, 3) a vacuolar nucleus with an enlarged nuclear body, 4) increase in nuclear division (2 mitoses/10 high power fields), 5) increase in nuclear to cytoplasmic ratio, and 6) pleomorphism. If one to two criteria are met, the granular cell tumor is considered to be atypical. The tumor is considered malignant if three or more criteria are met. Our patient did not have any of these criteria; hence, the diagnosis of benign granular cell tumor was made. In addition, clinical factors such as older patient age, larger tumor size, rapid growth, local recurrences and metastasis are all unfavorable prognostic factors [8].

This case highlights the fact that when patients present for 
their annual well-woman examination, clinicians should ask about vulva health as part of the usual gynecological screening. When vulva lesions are found, there should be a low threshold for biopsy. We suggest that when women present with a firm solitary lesion of the vulva, granular cell tumors should be among the differential diagnosis and the possibility of malignancy should not be excluded before histopathological evaluation and therefore wide local excision should be the treatment of choice.

\section{Grant Support}

None.

\section{References}

1. Committee opinion No. 534: well-woman visit. Obstet Gynecol. 2012;120(2 Pt 1):421-424.

2. ACOG Practice Bulletin No. 93: diagnosis and man- agement of vulvar skin disorders. Obstet Gynecol. 2008;111(5):1243-1253.

3. Jones RW, Joura EA. Analyzing prior clinical events at presentation in 102 women with vulvar carcinoma. Evidence of diagnostic delays. J Reprod Med. 1999;44(9):766-768.

4. Canavan TP, Cohen D. Vulvar cancer. Am Fam Physician. 2002;66(7):1269-1274.

5. Rivlin ME, Meeks GR, Ghafar MA, Lewin JR. Vulvar granular cell tumor. World J Clin Cases. 2013;1(4):149151.

6. Mehta V, Balachandran C, Rao L, Geeta V. Giant granular cell tumor of the vulva. Indian J Dermatol Venereol Leprol. 2010;76(3):263-265.

7. Fanburg-Smith JC, Meis-Kindblom JM, Fante R, Kindblom LG. Malignant granular cell tumor of soft tissue: diagnostic criteria and clinicopathologic correlation. Am J Surg Pathol. 1998;22(7):779-794.

8. Aoyama K, Kamio T, Hirano A, Seshimo A, Kameoka S. Granular cell tumors: a report of six cases. World J Surg Oncol. 2012;10:204. 